International Journal of Advanced Academic Research (Sciences, Technology and Engineering) | ISSN: 2488-9849 Vol. 6, Issue 8 (August, 2020)|www.ijaar.org

Journal DOI: 10.46654/ij.24889849

Article DOI: 10.46654/ij.24889849.e687

\title{
PHYSICAL AND MECHANICAL EVALUATION OF BALLAST AGGREGATES FROM SELECTED QUARRY SITES IN MPAPE, ABUJA, NIGERIA FOR RAILWAY TRACK CONSTRUCTION
}

\section{APEH OCHOLI ADEJOH SAMUEL ${ }^{1}$, APEH SUNDAY ${ }^{2 a}$, EGBUNU DANLADI ${ }^{2 b}$ AND THOMAS AKATA IBU ${ }^{2 c}$}

\author{
1,2a, 2b Department of Civil Engineering, Federal Polytechnic Idah, Kogi State, Nigeria. \\ 2c Department of Civil Engineering, Bayero University Kano, Nigeria.
}

Correspondence e-mail: engrsamapeh@gmail.com

\begin{abstract}
In this study, Samples of crushed aggregates were collected from six (6) Quarries, Hongyun Quarry, Arab Contractor Quarry, Perfect Stone Quarry, Exsamine Quarry I, Exsamine Quarry II, and Leenford Quarry in Mpape, Abuja, Nigeria.The physical and mechanical properties of the aggregates were carried out to assess their suitability for railway track construction. The results were then compared with the specifications as contained in British Standards. The study found that at Hongyun Quarry, Specific Gravity was 2.03, Water Absorption was 0.65\%, Moisture Content was 0.2\%, Flakiness Index was 21\%, Elongation Index was 35\%, Particle Length Index was 1.8\%, Aggregate crushing value was 16\%, Deval abrasion (wet \& dry) was $3 \%$, and Los angeles abrasion was 11\%, at Perfect Stone Quarry, the results obtained were 1.8, $0.64 \%, 0.40 \%, 26.7 \%, 38.3 \%, 3 \%, 11 \%, 2.3 \%$, and $12 \%$, at Arab Contractor quarry, these results were obtained; 1.92, 0.6\%, 0.42\%, 22\%, 26.3\%, 2.3\%, 16\%, 3.7\%, 11.3\%, at Exsamine quarry $I$, the results were $2.21,0.76 \%, 0.38 \%, 19.7 \%, 23.3 \%, 1.7 \%, 19 \%, 2.3 \%, 12 \%$, at Exsamine quarry II, the results were 2.23, 0.57\%, 0.43\%, 20.3\%, 33.7\%, 2.3\%, $12.7 \%, 3.7 \%$, $13 \%$, and at Leenford quarry, the result obtained were 2.19, 0.68\%, 0.28\%, 26.3\%, 29.3\%, $2.7 \%, 17.7 \%, 4.3 \%, 13.3 \%$. It was found that all the aggregate samples met the required limit for all the parameters tested. Therefore the study concluded that they are suitable to be used as Ballast in Railway Track Construction in Nigeria.
\end{abstract}

Keywords: Mpape, Physical and Mechanical Properties, Crushed Aggregates, Railway Track Construction, British Standards. 


\subsection{INTRODUCTION}

Nigeria is currently constructing new high-speed railway lines and old lines are undergoing rehabilitation across the country. Abuja, the nation's capital to Kaduna high-speed line has since been completed and been run. Lagos to Kano high-speed is currently under construction, having completed Lagos to Ibadan, the first segment on that line. The design of Lagos to Calabar through Port Harcourt is at advanced stage. All these were made possible through the 'PublicPrivate Partnership between Nigeria and Chinese Civil Engineering Construction Company, CCECC. It's a new era in Nigeria.

A huge quantity of aggregates is required for railway track construction. The engineering performance of crushed aggregates is strongly linked to their physical and mechanical properties (Anavind \& Animesh, 2005). The construction and rehabilitation of railway lines in Nigerian (Figures 1.1 and 1.2) need durable and cheap aggregates, and the properties of aggregates are very critical in the selection of good quality aggregates for use in the construction (Maatthew \& Rao, 2017). The purpose of this study is to assess the physical and mechanical properties of crushed stones and their suitability for use in civil engineering projects.

Traditionally, ballast has been used in rail track because it is relatively cheap and effective. Crushed gravel, limestone, basalt and granite have been used over the years because they possess the following characteristics: Hardness, Abrasion Resistance, Resistance to Weathering Action etc.

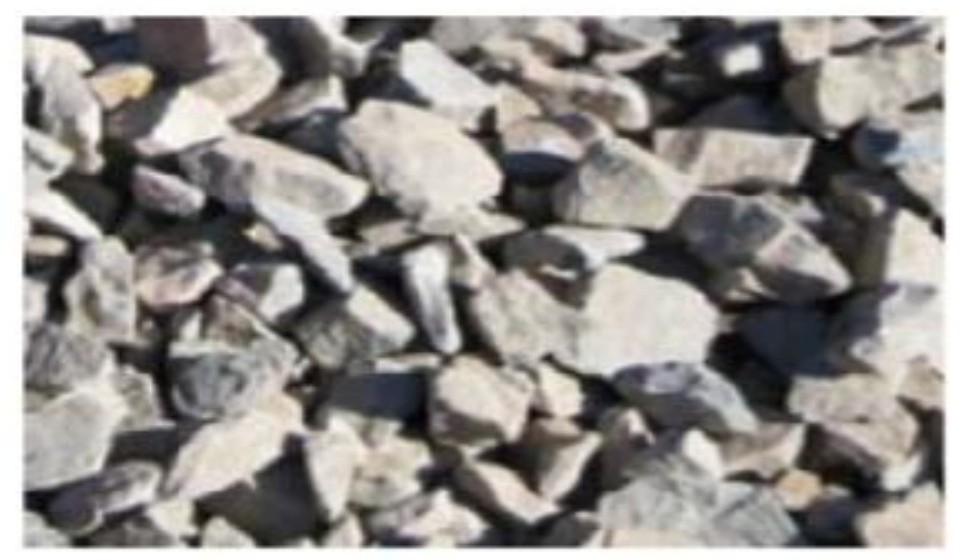

Figure 1.0: Well-graded ballast samples 


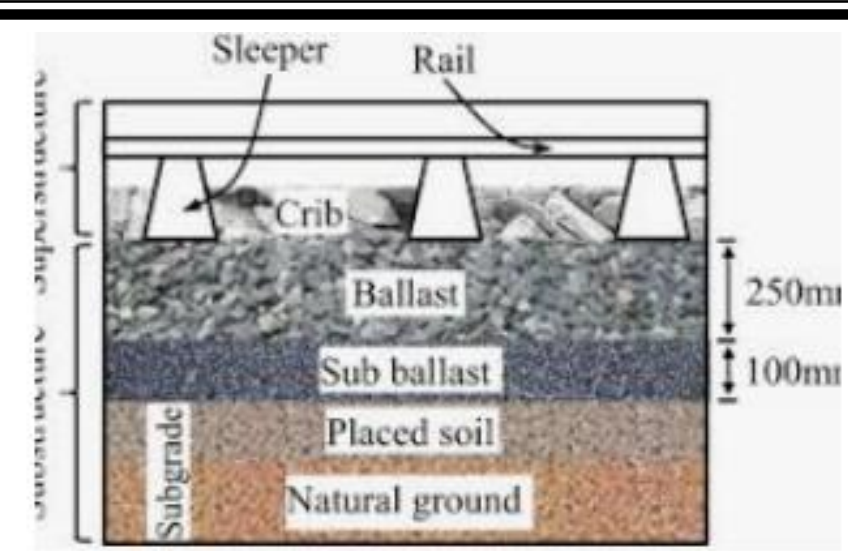

Figure 1.1: Ballasted Railway Track

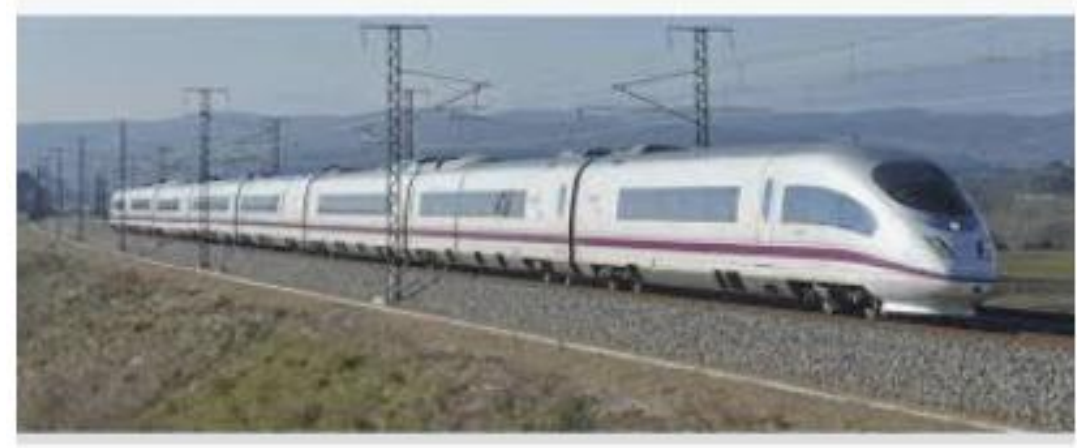

Figure 1.2: High-speed train travelling on ballasted track

\subsection{BALLAST SPECIFICATIONS}

Ideally, ballast particles used for rail track should be hard, durable, have good angularity, chemical resistance and be free from dust. Typical ballast size ranges from 20 to $50 \mathrm{~mm}$ in diameter and its performance is governed by the physical characteristics, though no single properties of ballast controls its performance. The aggregate size range used in this study is 31.5 $\mathrm{mm}$ to $50 \mathrm{~mm}$.

The United Kingdom follows the European railway ballast specification BS EN 13450 (2002). This specification requires the ballast to conform to the particle size distributions shown in the Table 1.0.

Table 1.0: Particle Size Distribution Specification (RT/CE/S/006, Issue 3, 200)

\begin{tabular}{|l|l|}
\hline Square Mesh Sieve (mm) & Cumulative \% by mass passing BS sieve \\
\hline 63 & 100 \\
\hline 50 & $70-100$ \\
\hline 40 & $30-65$ \\
\hline 31.5 & $0-25$ \\
\hline 22.4 & $0-3$ \\
\hline $32-50$ & $\geq 50$ \\
\hline
\end{tabular}




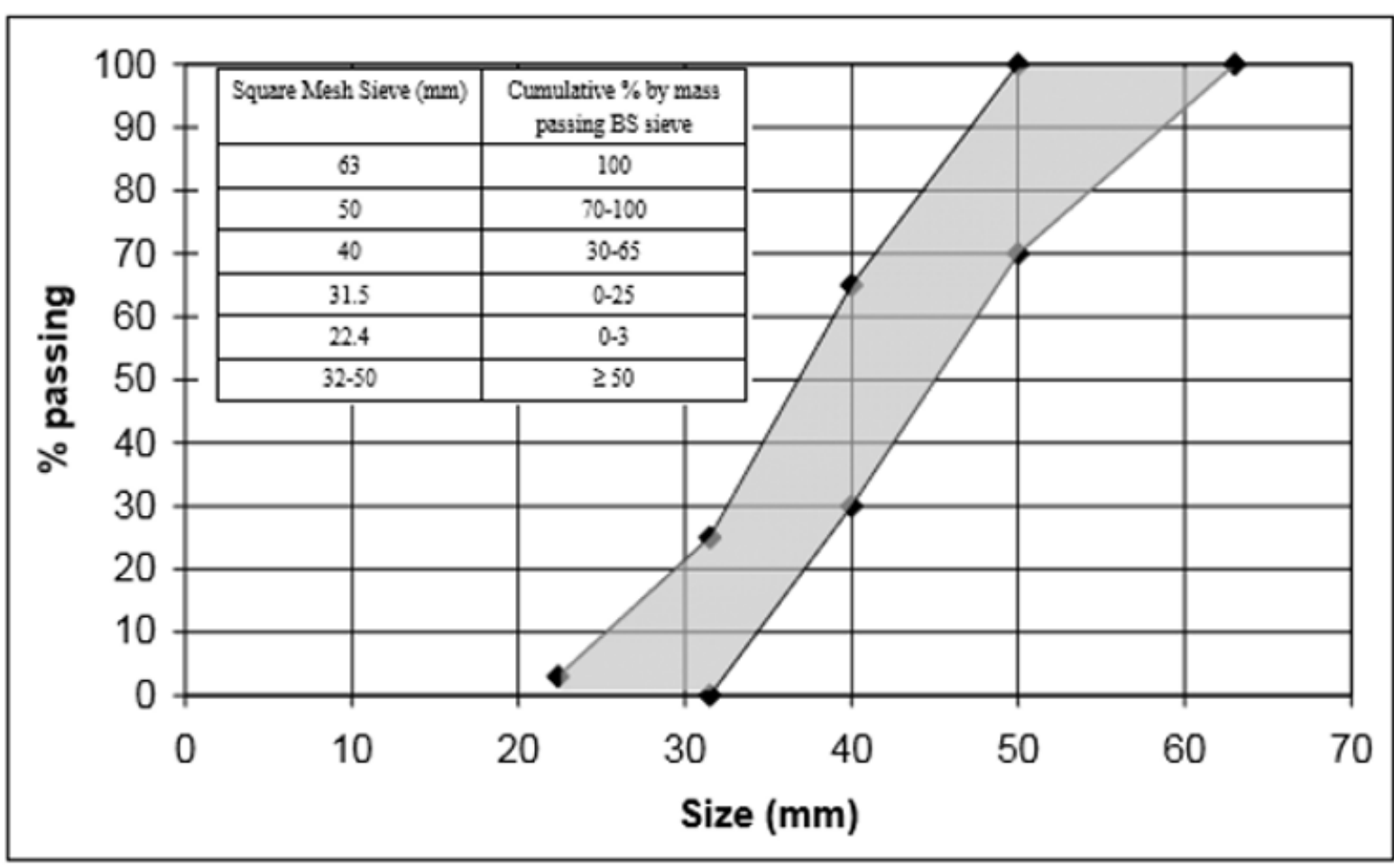

Figure 1.3: Specification for ballast particle size distribution

\subsection{BACKGROUND OF STUDY AREA}

Mpape is approximately 10 minutes' drive from the center of Abuja (Jimoh, 2017). It is one of the districts in Bwari Area Council of the Federal Capital Territory (FCT), Abuja. It lies on the foothills and on the top of the famous Mpape Rocks that are easily sighted from the neighbouring Maitama District (Dawam, 2000). Geographically, Mpape lies between Latitudes $9.175699^{\circ}$ and $9.113010^{\circ}$ north of the equator and Longitudes $7.463892^{\circ}$ and $7.524349^{\circ}$ east of the Greenwich Meridian (Figure 1). It occupies a land area of $44.325 \mathrm{Ha}$ and the largest slum settlement in Abuja and densely populated. The village has grown into an informal settlement with a population tethering over 1.1 million inhabitants due to urban-rural migration without adequate infrastructural facilities to cater for the population (Dawam, 2000). Mpape is surrounded by high grade metamorphism and igneous rocks, these rocks consist of gneiss, migmatites, granites and schist belt outcrops along the eastern margin of the area (FCDA, 1979, FCDA, 1998). The rocky nature of Mpape makes it suitable for quarry business and investors are doing well (Jimoh, 2017; Okeke, 2016).

The study discovered that nine quarry sites exist in Mpape, three have been abandoned while six are active. Out of the three that are located in the centre of Mpape, one is still active while two of them have been abandoned. The depth analysis revealed that the deepest site is $25 \mathrm{~m}$ at Julius Berger quarry site and the lowest depth was $6 \mathrm{~m}$ at Dantata quarry site. The study also revealed that the rocky surfaces has the highest elevation and are located in the upper and lower region of Mpape. The quarry sites and settlement are located on a high elevation ground. Furthermore, the study revealed that the derelict ponds found were formed because of rigorous quarry activities, since there are no tributaries connecting each of the derelict ponds. 


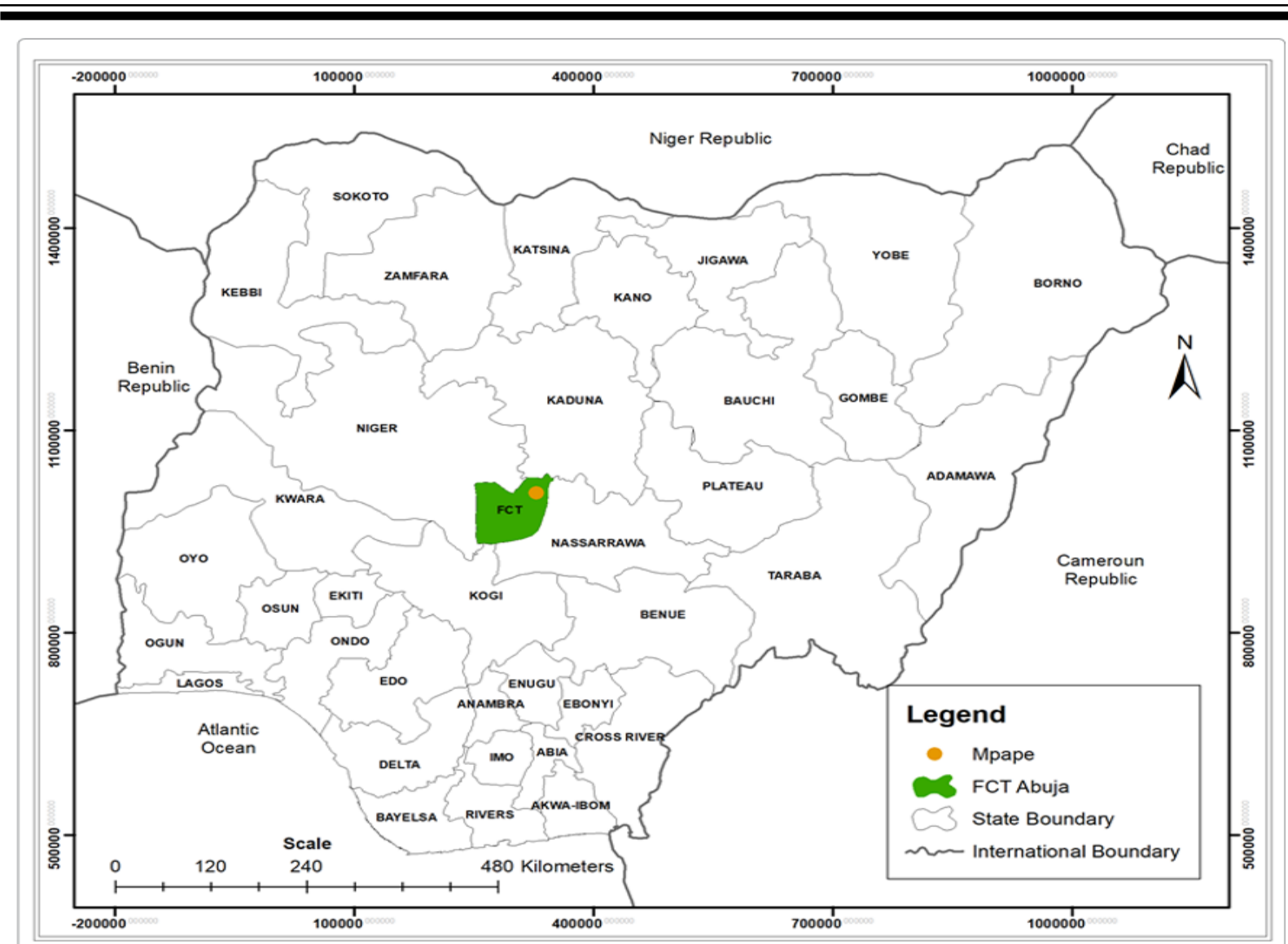

Figure 1.4: Nigeria Map Showing Mpape in FCT, Abuja (AGIS, 2017).

\subsection{STATEMENT OF PROBLEM}

The primary purpose of this study is to evaluate the physical and mechanical properties of crushed stones (granite) at Mpape, Abuja in Nigeria. This is to ensure that good quality aggregates are used to construct railway tracks across the country, because if poor quality aggregates are used, it will degrade and deform fairly quickly thereby increasing the cost of maintenance of the track-bed.

\subsection{MATERIALS AND METHODS}

\subsection{COLLECTION OF SAMPLE}

The materials for this study were collected from Mpape, Federal Capital Territory, Abuja, Nigeria. The sample sizes were between approximately $31.5 \mathrm{~mm}$ to $50 \mathrm{~mm}$.

Geographically, Mpape lies between Latitudes $9.175699^{\circ}$ and $9.113010^{\circ}$ north of the equator and Longitudes $7.463892^{\circ}$ and $7.524349^{\circ}$ east of the Greenwich Meridian. 


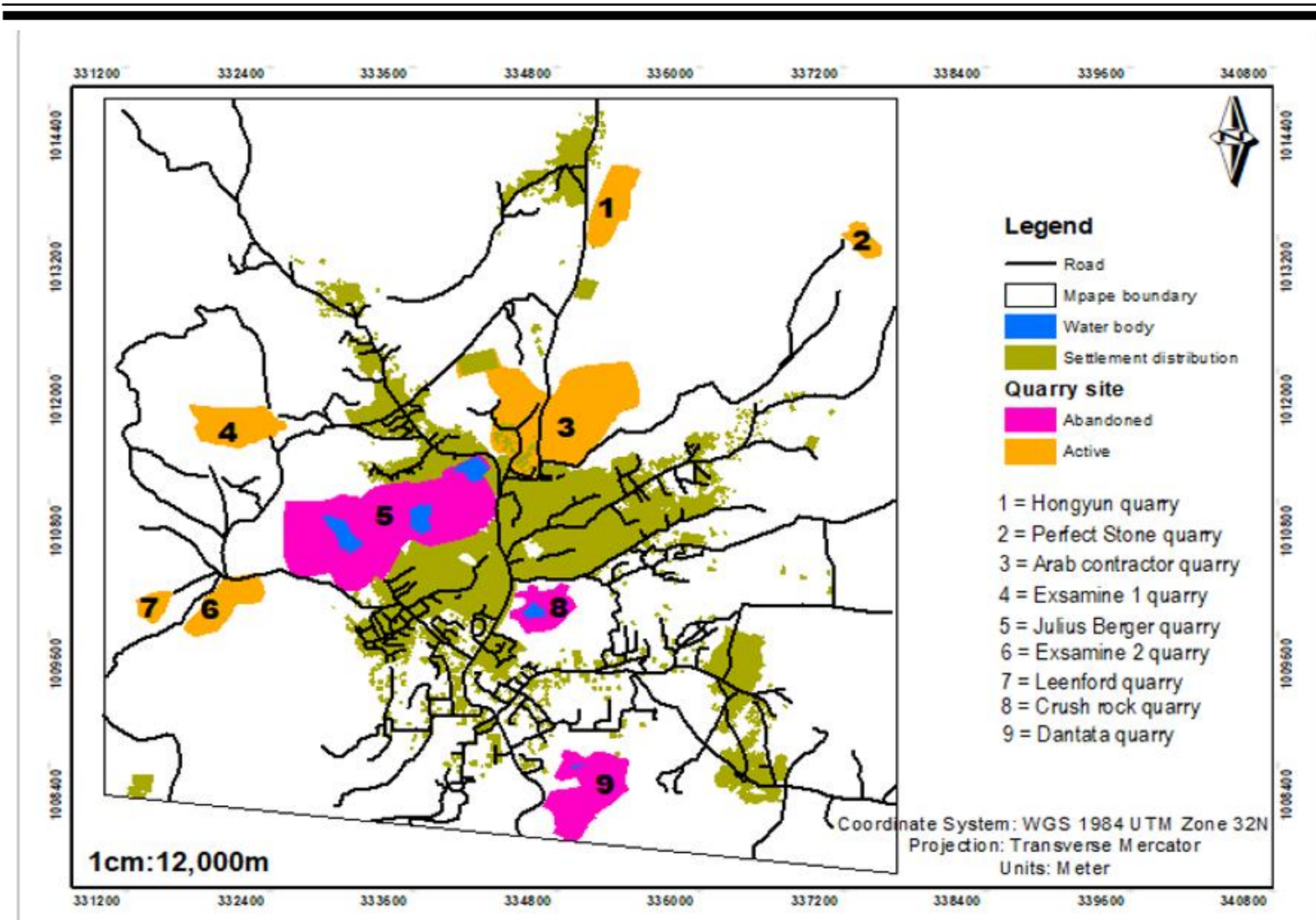

Figure 2.0: Distribution of quarry sites, settlements, roads and open-space, (Solomon et al. 2017).

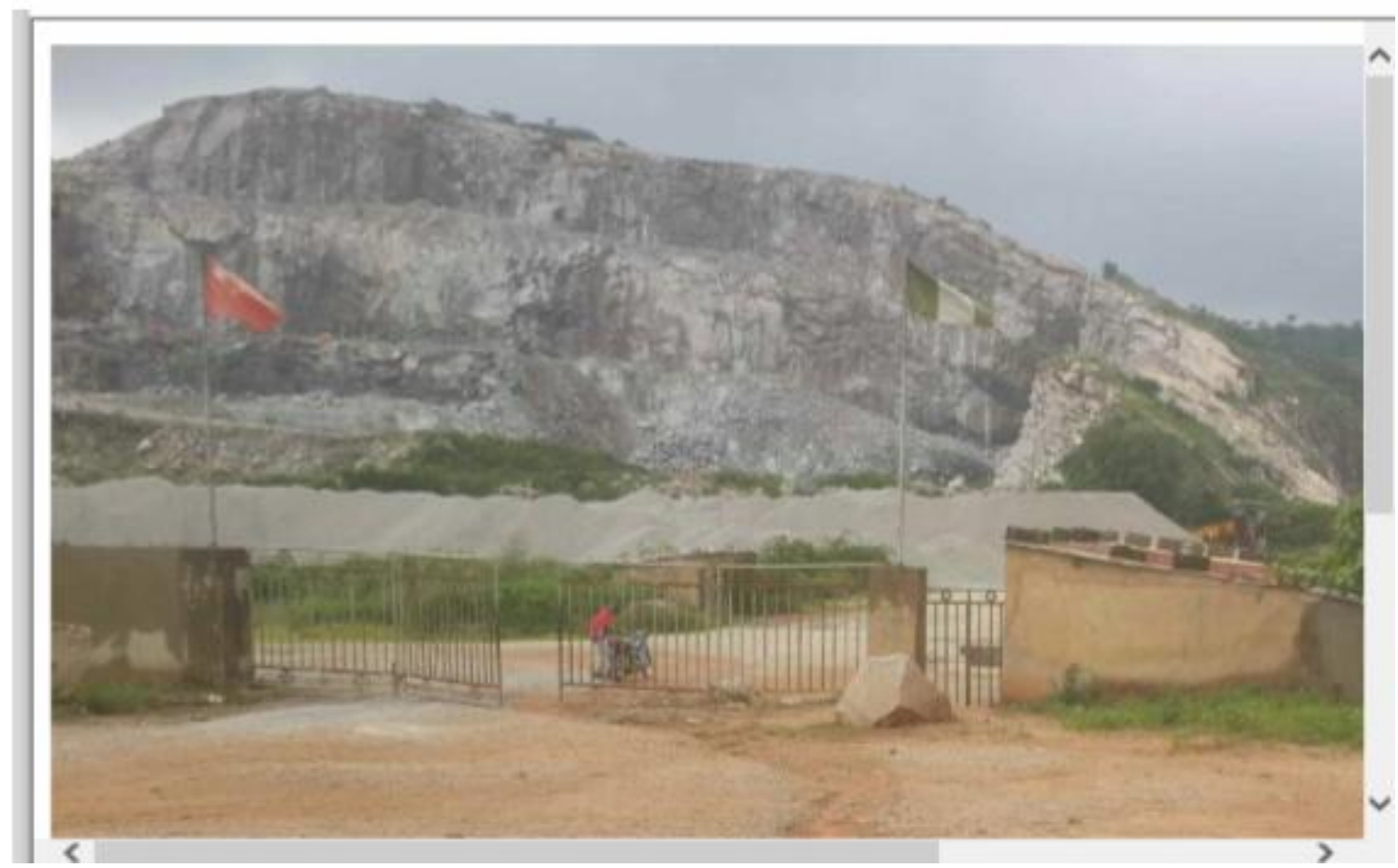

Figure 2.1: Hongyun Quarry Site, (Solomon et al., 2017). 


\subsection{METHODS}

To ensure that ballast is of good quality, it needs to be tested after the manufacturing process at the quarry, in order to ascertain their mechanical and dimensional properties. The ballast samples used were collected from six different quarries in Mpape, Abuja, Nigeria, measuring approximately 31.5 to $50 \mathrm{~mm}$, uniformly graded crushed hard stone which is durable, angular, equi-dimensional in shape and is almost non-flaky.

The six (6) selected quarries present a good representation of the type of rock that is obtainable within the area. The selections were made due to high patronage and the fact that production rate of aggregates from the quarries are high when compared with other quarries within Abuja, and the opinion of people sampled favoured Mpape quarries.

The sampling of aggregates for tests was in accordance to the sampling procedure in the British Standards. The samples were investigated in a well-equipped laboratory to determine the physical and mechanical characteristics of the aggregates. They were subjected to the following tests: Specific gravity Test, Water Absorption Test, Moisture content Test, Aggregates Impact Value Test, Aggregates Crushing Value Test, Flakiness Index Test, and Particle Length Index Test, in accordance to the relevant British Standards.

\subsection{RESULTS AND DISCUSSION}

\subsection{RESULTS}

Table 3.1: Physical and Mechanical Properties of Crushed Rock Obtained from Hongyun Quarry

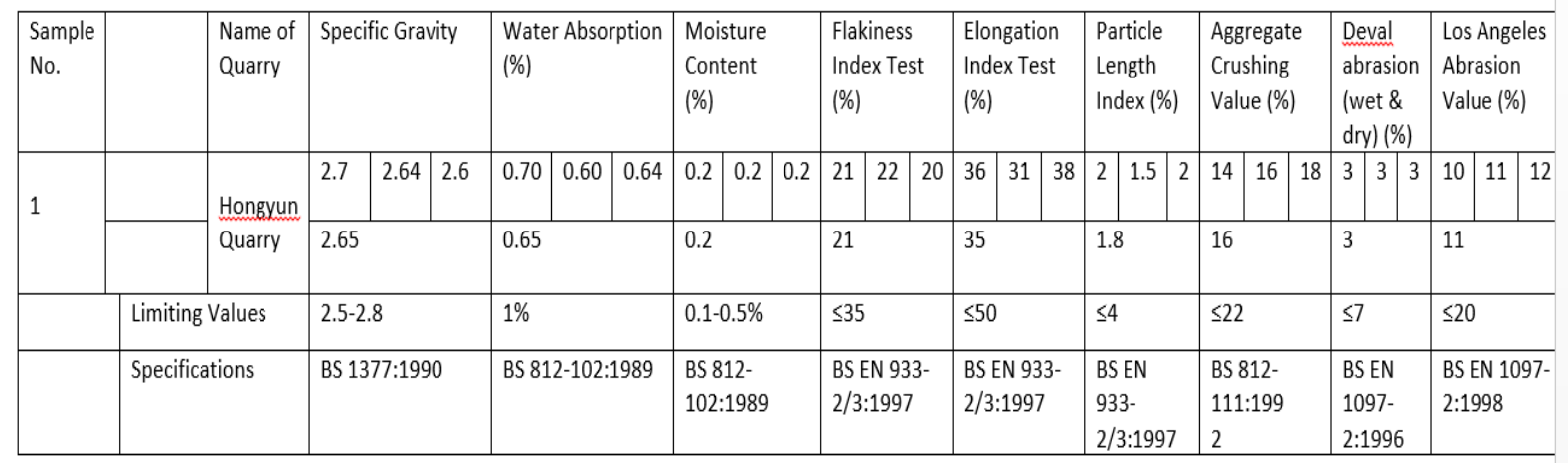


Table 3.2: Physical and Mechanical Properties of Crushed Aggregates Obtained from Perfect Stone Quarry

\begin{tabular}{|c|c|c|c|c|c|c|c|c|c|c|c|c|c|c|c|c|c|c|c|c|c|c|c|c|c|c|c|c|}
\hline \multirow{3}{*}{$\begin{array}{l}\begin{array}{l}\text { Sample } \\
\text { No. }\end{array} \\
2\end{array}$} & \multirow{3}{*}{$\begin{array}{l}\begin{array}{l}\text { Name } \\
\text { of } \\
\text { Quarry }\end{array} \\
\text { Perfect } \\
\text { Stone } \\
\text { Quarry }\end{array}$} & \multicolumn{3}{|c|}{ Specific Gravity } & \multicolumn{3}{|c|}{$\begin{array}{l}\text { Water Absorption } \\
(\%)\end{array}$} & \multicolumn{3}{|c|}{$\begin{array}{l}\text { Moisture Content } \\
\text { (\%) }\end{array}$} & \multicolumn{3}{|c|}{$\begin{array}{l}\text { Flakiness } \\
\text { Index Test } \\
\text { (\%) }\end{array}$} & \multicolumn{3}{|c|}{$\begin{array}{l}\text { Elongation } \\
\text { Index Test } \\
(\%)\end{array}$} & \multicolumn{3}{|c|}{$\begin{array}{l}\text { Particle } \\
\text { Length } \\
\text { Index } \\
(\%) \\
\end{array}$} & \multicolumn{3}{|c|}{\begin{tabular}{|l} 
Aggregate \\
Crushing \\
Value (\%)
\end{tabular}} & \multicolumn{3}{|c|}{$\begin{array}{l}\text { Deval } \\
\text { abrasion } \\
\text { (wet \& } \\
\text { dry) (\%) }\end{array}$} & \multicolumn{3}{|c|}{$\begin{array}{l}\text { Los Angeles } \\
\text { Abrasion } \\
\text { Value (\%) }\end{array}$} \\
\hline & & 2.59 & 2.43 & 2.4 & 0.60 & 0.61 & 0.72 & 0.40 & 0.41 & 0.38 & 23 & 27 & 30 & 41 & 38 & 36 & 3 & 3 & 3 & 12 & 10 & 11 & 3 & 2 & 2 & 12 & 12 & 12 \\
\hline & & \multicolumn{3}{|l|}{2.47} & \multicolumn{3}{|l|}{0.64} & \multicolumn{3}{|l|}{0.40} & \multicolumn{3}{|c|}{26.7} & \multicolumn{3}{|c|}{38.3} & \multicolumn{3}{|l|}{3} & \multicolumn{3}{|c|}{11} & \multicolumn{3}{|c|}{2.3} & \multicolumn{3}{|l|}{12} \\
\hline \multicolumn{2}{|c|}{ Limiting Values } & \multicolumn{3}{|c|}{$2.5-2.8$} & \multicolumn{3}{|l|}{$1 \%$} & \multicolumn{3}{|c|}{$0.1-0.5 \%$} & \multicolumn{3}{|c|}{$\leq 35$} & \multicolumn{3}{|c|}{$\leq 50$} & \multicolumn{3}{|c|}{$\leq 4$} & \multicolumn{3}{|c|}{$\leq 22$} & \multicolumn{3}{|c|}{$\leq 7$} & \multicolumn{3}{|c|}{$\leq 20$} \\
\hline \multicolumn{2}{|c|}{ Specifications } & \multicolumn{3}{|c|}{ BS 1377:1990 } & BS 81 & $2-102$ & 1989 & BS 81 & $2-102$ & 1989 & & $\begin{array}{l}\mathrm{N} 9 \\
199\end{array}$ & & $\begin{array}{l}\text { BS } \\
2 / 3\end{array}$ & $\begin{array}{l}\mathrm{EN} \mathrm{9} \\
199\end{array}$ & & & $\mathrm{~N}$ & & $\begin{array}{l}\text { BS } \\
111\end{array}$ & $\begin{array}{l}312- \\
: 199\end{array}$ & & & & & & $\begin{array}{l}\mathrm{N} 10 \\
98\end{array}$ & \\
\hline
\end{tabular}

Table 3.3: Physical and Mechanical Properties of Crushed Aggregates Obtained from Arab Contractor Quarry

\begin{tabular}{|c|c|c|c|c|c|c|c|c|c|c|c|c|c|c|c|c|c|c|c|c|c|c|c|c|c|c|c|c|}
\hline $\begin{array}{l}\text { Sample } \\
\text { No. }\end{array}$ & $\begin{array}{l}\text { Name of } \\
\text { Quarry }\end{array}$ & Speci & ic Gra & & $\begin{array}{l}\text { Wate } \\
(\%)\end{array}$ & Absor & ption & $\begin{array}{l}\text { Moist } \\
\text { (\%) }\end{array}$ & ure Co & ntent & $\begin{array}{l}\text { Flak } \\
\text { Inde } \\
\text { (\%) }\end{array}$ & $\begin{array}{l}\text { ines } \\
\text { ex Te }\end{array}$ & & $\begin{array}{l}\text { Elo } \\
\text { Ind } \\
\text { (\%) }\end{array}$ & $\begin{array}{l}\text { gati } \\
\times \mathrm{Te}\end{array}$ & & $\begin{array}{l}\text { Par } \\
\text { Ler } \\
\text { Ind } \\
(\%)\end{array}$ & $\begin{array}{l}\text { rticle } \\
\text { igth } \\
\text { lex }\end{array}$ & & $\begin{array}{l}\text { Aggr } \\
\text { Crus } \\
\text { Valu }\end{array}$ & $\begin{array}{l}\text { regat } \\
\text { shing } \\
\text { ue (\%) }\end{array}$ & & $\begin{array}{l}\text { Des } \\
\text { Abr } \\
\text { (we } \\
\text { dry }\end{array}$ & $\begin{array}{l}\text { al } \\
\text { asio } \\
\text { t \& } \\
\text { (\%) }\end{array}$ & & $\begin{array}{l}\text { os } \\
\text { Abr } \\
\text { Valu }\end{array}$ & $\begin{array}{l}\text { Ange } \\
\text { asion } \\
\text { e (\% }\end{array}$ & \\
\hline \multirow{2}{*}{3} & \multirow{2}{*}{$\begin{array}{l}\text { Arab } \\
\text { Contractor } \\
\text { Quarry }\end{array}$} & 2.75 & 2.73 & 2.64 & 0.50 & 0.61 & 0.68 & 0.42 & 0.46 & 0.39 & 22 & 23 & 21 & 26 & 29 & 24 & 3 & 2 & 2 & 16 & 18 & 14 & 3 & 4 & & 10 & 12 & 12 \\
\hline & & \multicolumn{3}{|l|}{2.71} & \multicolumn{3}{|l|}{0.60} & \multicolumn{3}{|l|}{0.42} & \multicolumn{3}{|l|}{22} & \multicolumn{3}{|c|}{26.3} & \multicolumn{3}{|c|}{2.3} & \multicolumn{3}{|l|}{16} & \multicolumn{2}{|c|}{3.7} & \multicolumn{4}{|c|}{11.3} \\
\hline \multicolumn{2}{|c|}{ Limiting Values } & \multicolumn{3}{|c|}{$2.5-2.8$} & \multicolumn{3}{|l|}{$1 \%$} & \multicolumn{3}{|c|}{$0.1-0.5 \%$} & \multicolumn{3}{|l|}{$\leq 35$} & \multicolumn{3}{|c|}{$\leq 50$} & \multicolumn{3}{|l|}{$\leq 4$} & \multicolumn{3}{|l|}{$\leq 22$} & \multicolumn{2}{|l|}{$\leq 7$} & \multicolumn{4}{|c|}{$\leq 20$} \\
\hline \multicolumn{2}{|c|}{ Specifications } & \multicolumn{3}{|c|}{ BS 1377:1990 } & \multicolumn{3}{|c|}{ BS 812-102:1989 } & \multicolumn{3}{|c|}{ BS 812-102:1989 } & \multicolumn{3}{|c|}{$\begin{array}{l}\text { BS EN 933- } \\
2 / 3: 1997\end{array}$} & \multicolumn{3}{|c|}{$\begin{array}{l}\text { BS EN 933- } \\
2 / 3: 1997\end{array}$} & \multicolumn{3}{|c|}{$\begin{array}{l}\text { BS EN } \\
933- \\
2 / 3: 1997\end{array}$} & \multicolumn{3}{|c|}{$\begin{array}{l}\text { BS 812- } \\
\text { 111:1992 }\end{array}$} & \multicolumn{2}{|c|}{$\begin{array}{l}\text { BS EN } \\
1097- \\
2: 1996\end{array}$} & \multicolumn{4}{|c|}{$\begin{array}{l}\text { BS EN 1097- } \\
\text { 2:1998 }\end{array}$} \\
\hline
\end{tabular}

Table 3.4: Physical and Mechanical Properties of Crushed Aggregates Obtained from Exsamine Quarry I

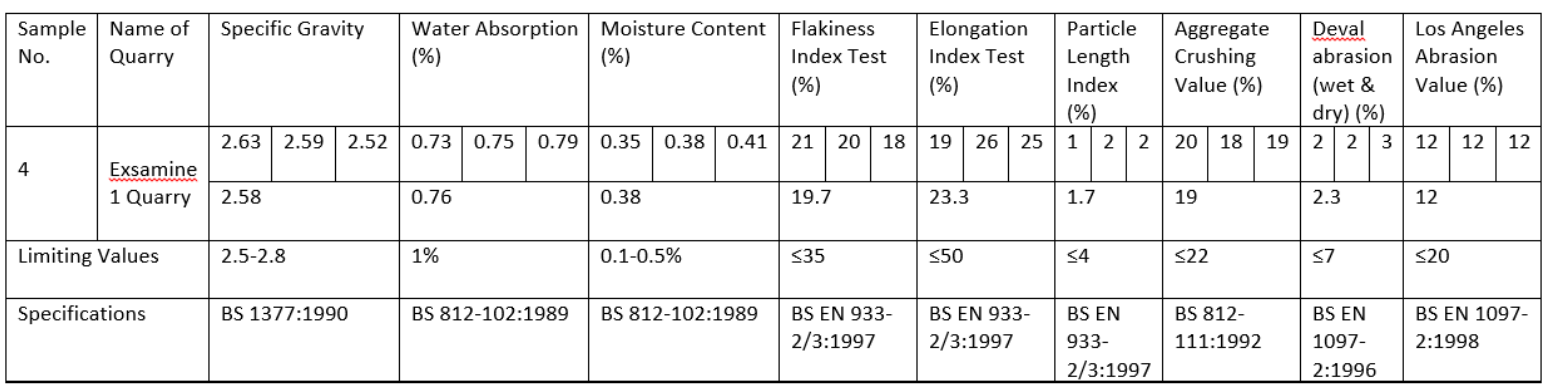

Table 3.5: Physical and Mechanical Properties of Crushed Aggregates Obtained from Exsamine Quarry II

\begin{tabular}{|c|c|c|c|c|c|c|c|c|c|c|c|c|c|c|c|c|c|c|c|c|c|c|c|c|c|c|}
\hline $\begin{array}{l}\text { Sample } \\
\text { No. }\end{array}$ & $\begin{array}{l}\text { Name of } \\
\text { Quarry }\end{array}$ & Speci & ic Grav & & $\begin{array}{l}\text { Wate } \\
(\%)\end{array}$ & Absor & ption & $\begin{array}{l}\text { Moist } \\
(\%)\end{array}$ & ure Co & tent & $\begin{array}{l}\text { Flak } \\
\text { Inde } \\
\text { (\%) }\end{array}$ & $\begin{array}{l}\text { ness } \\
\times \text { Tes }\end{array}$ & & $\begin{array}{l}\text { Elon } \\
\text { Inde } \\
\text { (\%) }\end{array}$ & $\begin{array}{l}\text { gatic } \\
x \text { Tes }\end{array}$ & & $\begin{array}{l}\text { Part } \\
\text { Leng } \\
\text { Inde } \\
\text { (\%) }\end{array}$ & & $\begin{array}{l}\text { Agg } \\
\text { Cru } \\
\text { Valu }\end{array}$ & $\begin{array}{l}\text { regat } \\
\text { sing } \\
\text { e (\%) }\end{array}$ & & $\begin{array}{l}\text { Deval } \\
\text { abras } \\
\text { (wet } \\
\text { dry) ( }\end{array}$ & & $\begin{array}{l}\text { Los } \\
\text { Abr } \\
\text { Valu }\end{array}$ & $\begin{array}{l}\text { Ange } \\
\text { sion } \\
\text { e (\%) }\end{array}$ & \\
\hline \multirow{2}{*}{5} & \multirow{2}{*}{$\begin{array}{l}\text { Exsamine } \\
2 \text { Quarry }\end{array}$} & 2.71 & 2.64 & 2.62 & 0.51 & 0.57 & 0.64 & 0.43 & 0.47 & 0.39 & 17 & 20 & 24 & 32 & 36 & 33 & 2 & $\begin{array}{ll}2 & 3\end{array}$ & 13 & 13 & 12 & \begin{tabular}{l|l}
3 & 4
\end{tabular} & 4 & 14 & 12 & 13 \\
\hline & & \multicolumn{3}{|l|}{2.66} & \multicolumn{3}{|l|}{0.57} & \multicolumn{3}{|l|}{0.43} & \multicolumn{3}{|c|}{20.3} & \multicolumn{3}{|c|}{33.7} & \multicolumn{2}{|l|}{2.3} & \multicolumn{3}{|c|}{12.7} & \multicolumn{2}{|l|}{3.7} & \multicolumn{3}{|l|}{13} \\
\hline \multicolumn{2}{|c|}{ Limiting Values } & \multicolumn{3}{|c|}{$2.5-2.8$} & \multicolumn{3}{|l|}{$1 \%$} & \multicolumn{3}{|c|}{$0.1-0.5 \%$} & \multicolumn{3}{|l|}{$\leq 35$} & \multicolumn{3}{|l|}{$\leq 50$} & \multicolumn{2}{|l|}{$\leq 4$} & \multicolumn{3}{|l|}{$\leq 22$} & \multicolumn{2}{|l|}{$\leq 7$} & \multicolumn{3}{|l|}{$\leq 20$} \\
\hline \multicolumn{2}{|c|}{ Specifications } & \multicolumn{3}{|c|}{ BS 1377:1990 } & \multicolumn{3}{|c|}{ BS 812-102:1989 } & \multicolumn{3}{|c|}{ BS 812-102:1989 } & \multicolumn{3}{|c|}{$\begin{array}{l}\text { BS EN 933- } \\
2 / 3: 1997\end{array}$} & \multicolumn{3}{|c|}{$\begin{array}{l}\text { BS EN 933- } \\
2 / 3: 1997\end{array}$} & \multicolumn{2}{|c|}{$\begin{array}{l}\text { BS EN } \\
933- \\
2 / 3 \cdot 1997\end{array}$} & \multicolumn{3}{|c|}{$\begin{array}{l}\text { BS 812- } \\
111: 1992\end{array}$} & \multicolumn{2}{|c|}{$\begin{array}{l}\text { BS EN } \\
1097- \\
2 \cdot 1996\end{array}$} & \multicolumn{3}{|c|}{$\begin{array}{l}\text { BS EN 1097- } \\
2: 1998\end{array}$} \\
\hline
\end{tabular}


International Journal of Advanced Academic Research (Sciences, Technology and Engineering) | ISSN: 2488-9849 Vol. 6, Issue 8 (August, 2020)|www.ijaar.org

Journal DOI: 10.46654/ij.24889849

Article DOI: 10.46654/ij.24889849.e687

Table 3.6: Physical and Mechanical Properties of Crushed Aggregates Obtained from Leenford Quarry

\begin{tabular}{|c|c|c|c|c|c|c|c|c|c|c|c|c|c|c|c|c|c|c|c|c|c|c|c|c|c|c|c|c|}
\hline \multirow{3}{*}{$\begin{array}{l}\text { Sample } \\
\text { No. } \\
6\end{array}$} & \multirow{3}{*}{$\begin{array}{l}\text { Name of } \\
\text { Quarry } \\
\text { Leenford } \\
\text { Quarry }\end{array}$} & \multicolumn{3}{|c|}{ Specific Gravity } & \multicolumn{3}{|c|}{$\begin{array}{l}\text { Water Absorption } \\
(\%)\end{array}$} & \multicolumn{3}{|c|}{$\begin{array}{l}\text { Moisture Content } \\
(\%)\end{array}$} & \multicolumn{3}{|c|}{$\begin{array}{l}\text { Flakiness } \\
\text { Index Test } \\
(\%)\end{array}$} & \multicolumn{3}{|c|}{$\begin{array}{l}\text { Elongation } \\
\text { Index Test } \\
(\%)\end{array}$} & \multicolumn{3}{|c|}{$\begin{array}{l}\text { Particle } \\
\text { Length } \\
\text { Index } \\
(\%) \\
\end{array}$} & \multicolumn{3}{|c|}{$\begin{array}{l}\text { Aggregate } \\
\text { Crushing } \\
\text { Value (\%) }\end{array}$} & \multicolumn{3}{|c|}{$\begin{array}{l}\text { Deval } \\
\text { abrasion } \\
\text { (wet \& } \\
\text { dry) (\%) }\end{array}$} & \multicolumn{3}{|c|}{$\begin{array}{l}\text { Los Angeles } \\
\text { Abrasion } \\
\text { Value (\%) }\end{array}$} \\
\hline & & 2.67 & 2.52 & 2.50 & 0.67 & 0.68 & 0.70 & 0.20 & 0.31 & 0.34 & 26 & 23 & 30 & 31 & 29 & 28 & 2 & 3 & 3 & 15 & 15 & 23 & 4 & 4 & 5 & 13 & 14 & 13 \\
\hline & & \multicolumn{3}{|l|}{2.56} & \multicolumn{3}{|l|}{0.68} & \multicolumn{3}{|l|}{0.28} & \multicolumn{3}{|c|}{26.3} & \multicolumn{3}{|c|}{29.3} & \multicolumn{3}{|c|}{2.7} & \multicolumn{3}{|c|}{17.7} & \multicolumn{3}{|c|}{4.3} & \multicolumn{3}{|c|}{13.3} \\
\hline \multicolumn{2}{|c|}{ Limiting Values } & \multicolumn{3}{|c|}{$2.5-2.8$} & \multicolumn{3}{|l|}{$1 \%$} & \multicolumn{3}{|c|}{$0.1-0.5 \%$} & \multicolumn{3}{|c|}{$\leq 35$} & \multicolumn{3}{|c|}{$\leq 50$} & \multicolumn{3}{|l|}{$\leq 4$} & \multicolumn{3}{|c|}{$\leq 22$} & \multicolumn{3}{|l|}{$\leq 7$} & \multicolumn{3}{|c|}{$\leq 20$} \\
\hline \multicolumn{2}{|c|}{ specifications } & \multicolumn{3}{|c|}{ BS 1377:1990 } & $\begin{array}{l}\text { BS EI } \\
: 198 \mathrm{~s}\end{array}$ & $933-1$ & & $\begin{array}{l}\text { BS EN } \\
: 1989\end{array}$ & $933-1$ & & $\begin{array}{l}\text { BSE } \\
2 / 3\end{array}$ & : 1997 & & $\begin{array}{l}\mathrm{BS} \\
2 / 3\end{array}$ & :N 93 & & $\begin{array}{l}\text { BS } \\
93 \\
2 / 3\end{array}$ & 199: & & $\begin{array}{l}\text { BS \& } \\
111\end{array}$ & $\begin{array}{l}12- \\
1992\end{array}$ & & & & & & & $97-$ \\
\hline
\end{tabular}

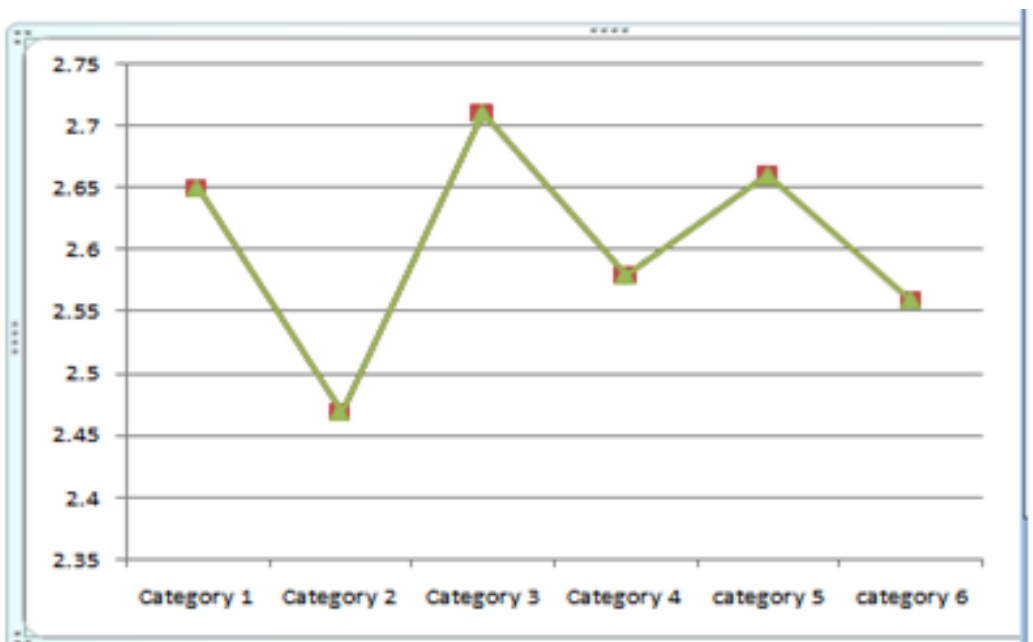

Figure 3.1: Graph of Specific gravities of samples collected from six quarry sites

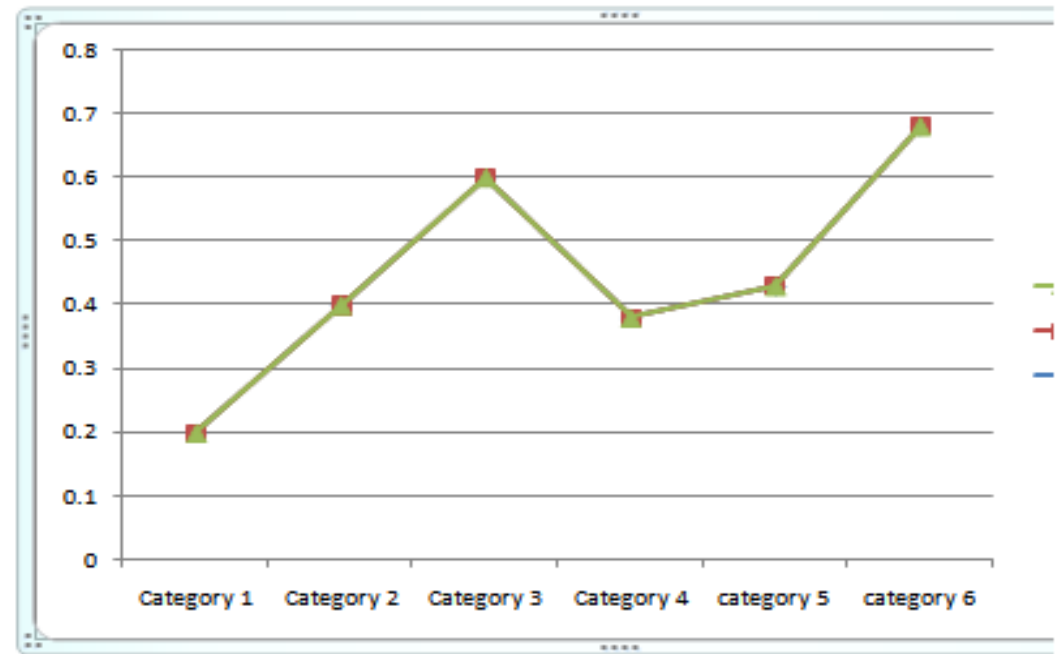

Figure 3.2: Graph of water absorption of samples collected from six quarry sites 


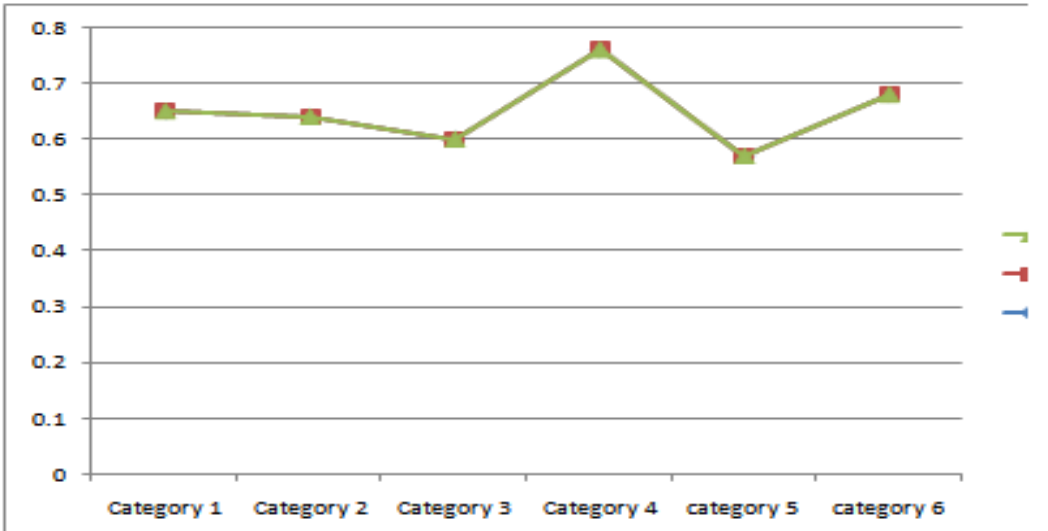

Figure 3.3: Graph of Moisture Content of samples collected from six quarry sites

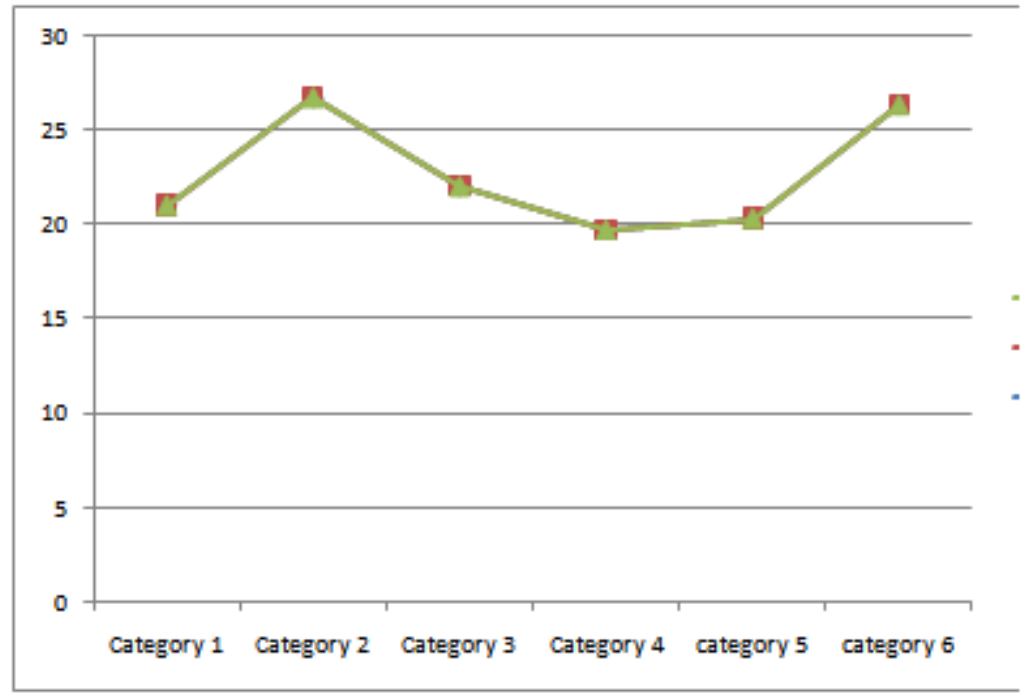

Figure 3.4: Graph of Flakiness Index of samples collected from six quarry sites 


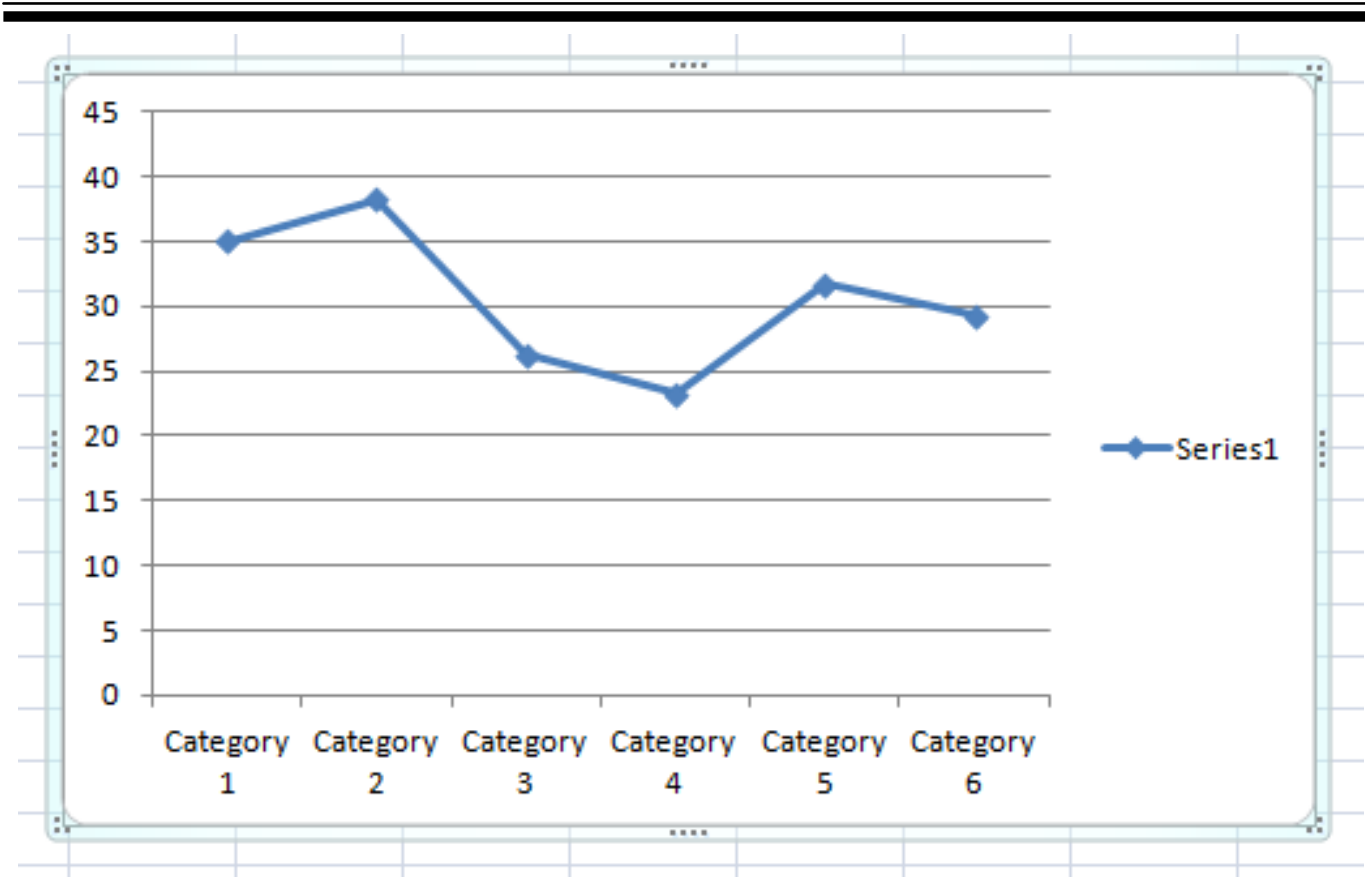

Figure 3.5: Graph of Elongation Index of samples collected from six quarry sites

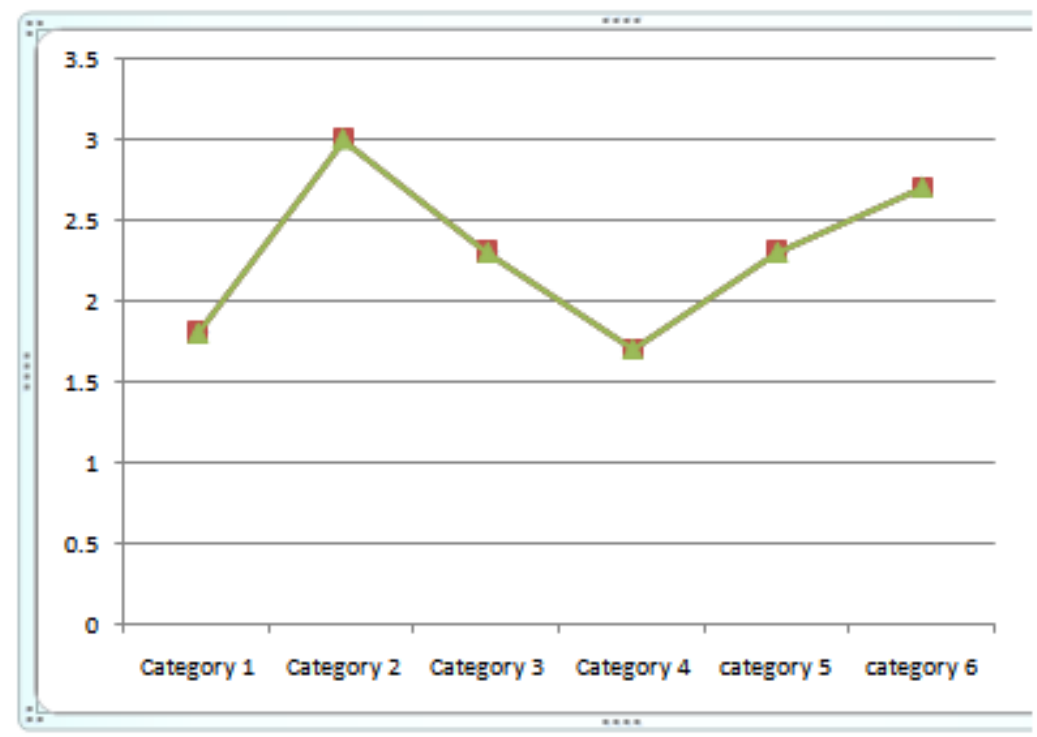

Figure 3.6: Graph of Particle Length Index of samples collected from six quarry sites 


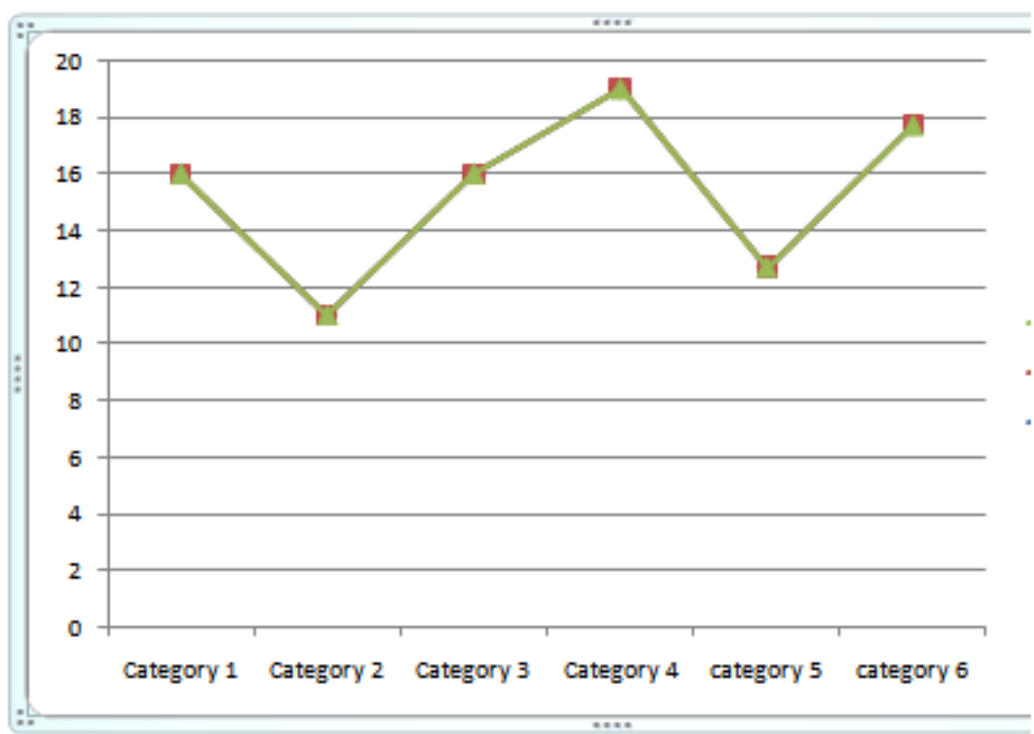

Figure 3.7: Graph of Aggregate Crushing Value of samples collected from six quarry sites

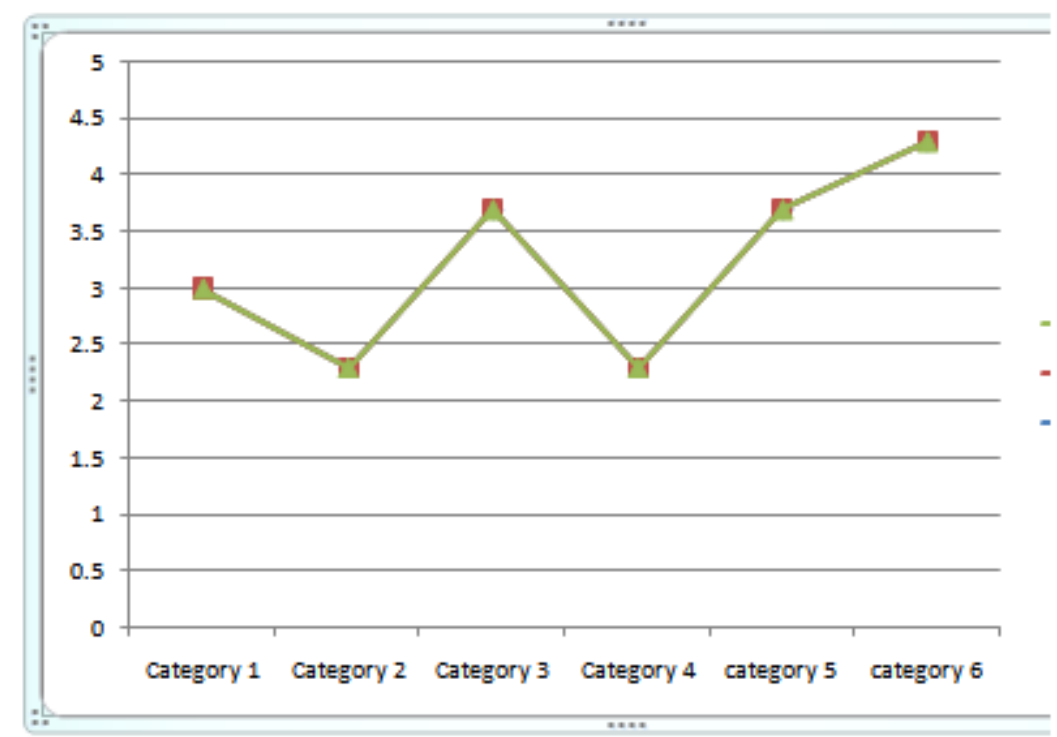

Figure 3.8: Graph of Deval Abrasion (wet \& dry) of samples collected from six quarry sites 


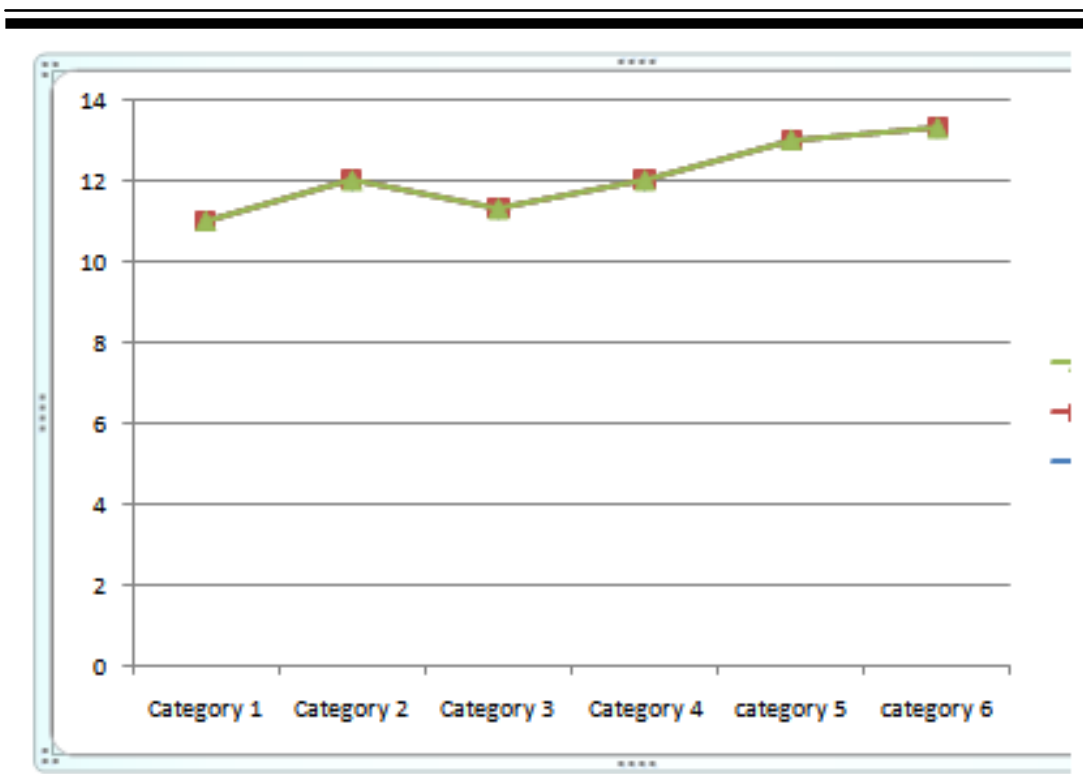

Figure 3.9: Graph of Los Angeles Abrasion of samples collected from six quarry sites

Where,

Category 1- Hongyun Quarry

Category 2 - Perfect Stone Quarry

Category 3 - Arab Contractor Quarry

Category 4 - Exsamine Quarry 1

Category 5 - Exsamine Quarry 2

Category 6 - Leenford Quarry

\subsection{DISCUSSSION}

The results of the Specific Gravity Values (Figure 3.1) range from 2.47-2.71. Perfect stone Quarry had the least specific gravity value of 2.47, while Arab contractor had the highest value of 2.71. However, all of the value still fell within the recommended value except that of Perfect stone which fell slightly below the recommended value.

Water Absorption Values (Figure 3.2) fell between 0.57-0.76\%. The least value of $0.57 \%$ was obtained at Exsamine Quarry II, while the highest value of $0.76 \%$ was obtained at Exsamine Quarry II. However, all the values did not exceed the limiting value of $1 \%$.

Moisture Content Values (Figure 3.3) obtained were between $0.20-0.43 \%$. The least value of $0.20 \%$ was obtained at Hongyun Quarry, while the highest value of $0.43 \%$ was obtained at Exsamine Quarry II. However, all the values were within the recommended value.

Flakiness Index Test Values (Figure 3.4) were found to be between 19.7-26.7\%. The lowest value of $19.7 \%$ was obtained at Exsamine quarry I, while the highest value of $26.7 \%$ was obtained at Perfect stone quarry. However, all the values were within the specified value of $\leq 35$. 
Elongation Index Test Values (Figure 3.5) range from 23.3-38.3\%. The lowest value of 23.3\% was recorded at Examine quarry I, while the highest value of $38.3 \%$ was recorded at Perfect stone quarry. However, all the value were within the recommended value.

Particle Length Index Test Values (Figure 3.6) range from 1.7-2.7\%. The lowest value of $1.7 \%$ was recorded at Exsamine quarry I, while the highest value of $2.7 \%$ was recorded at Leenford quarry

Aggregate Crushing Values (Figure 3.7) range from 11-19\%. The lowest value of 11\% was recorded at Exsamine quarry I, while the highest value of $19 \%$ was recorded at Perfect stone quarry. However, all of the values fell within the recommended value.

Deval Abrasion (wet and dry) values (Figure 3.8) range from 2.3-4.3\%. The lowest value of $2.3 \%$ were recorded at Perfect stone quarry and Exsamine quarry I, while the highest value of $4.3 \%$ was recorded at Leenford quarry. However, all values recorded fell within the recommended value.

Los Angeles Abrasion values (Figure 3.9) range from 11-13.3\%. The least value of $11 \%$ was recorded at Hongyun quarry, while the highest value of $13.3 \%$ was recorded at Hongyun quarry. However, all values fell within the recommended value.

\subsection{CONCLUSION AND RECOMMENDATION}

\subsection{CONCLUSION}

The results of the assessment on crushed aggregates from six (6) selected quarries located at Mpape, Abuja, Nigeria showed that the values of all the parameters obtained on samples at Hongyun quarry, Perfect Stone quarry, Arab Contractor quarry, Exsamine quarry I, Exsamine quarry II, and Leenford quarry fall within the specified ranges as recommended by British Standards of aggregate stone production for both flexible and rigid pavement construction. Therefore, the study concluded that the crushed stones obtained are suitable for use in Railway Track Construction in Nigeria.

\subsection{RECOMMENDATION}

The study recommends that all the aggregate samples collected from six quarry sites at Mpape in Abuja, Nigeria are fit to be used as ballast in railway track construction in Nigeria, since they have satisfied both the physical and mechanical properties requirements. 


\section{REFERENCES}

AGIS (2017). Abuja Geographic Information Systems 2: 1.

Aravind, K.,Animesh, D. (2005). Industrial Waste in Highway Construction.Department of Civil Engineering, IIT Kanpur. 1-4. http://home.iitk.ac.in/ adas/article07. Pdf.

British Standard: BS EN 13450 (2002). “Aggregates for Railway Ballast”.

British Standard: BS EN 1097-6 (2000). Determination of particle density and water absorption.

British Standard: BS EN 1097-2. (1998). Tests for mechanical and physical properties of aggregates- Methods for the determination of resistance to fragmentation.

British Standard: BS EN 933-3. (1997). Determination of particle shape- Flakiness index.

British Standard: BS EN 1097-2. (1996). Tests for mechanical and physical properties of aggregates- Determination of the resistance to wear (micro Deval).

British Standard: BS 812-111 (1990). "Methods for Determination of Aggregate Crushing Value (ACV)".

British Standard Institute BS 812-105.1: 1989. Methods for determination of particle shape Section 105.1 Flakiness index.

British Standard: BS 812-103.1 (1985). "Methods for Determination of Particle Size Distribution- Sieve Tests".

Dawam P.D. (2000). The geography of Abuja federal capital territory. Famous/ Asanlu Publishers, Minna, Nigeria.

Federal Capital Development Authority (FCDA) (1979). The master plan for Abuja the new federal capital of Nigeria. FCDA, Abuja, Nigeria.

Federal Capital Development Authority (FCDA) (1998). Abuja Handbook. FCDA, Abuja, Nigeria.

Jimoh I. (2017). The Abuja inquirer. Mpape: A tale of modernity and slum 2: 1.

Mathew, T.V.,Rao, K.V. (2007). Introduction to Transportation Engineering.Chapter 22. Pavement Materials: Aggregates

Okeke, J. (2016). The authority newspaper. Mpape: The Good, the Bad and the Ugly.

Solomon, M, Cyril, K.E, Taiye, O, and Mwanret G.D. (2017). Spatial-Temporal Assessment of Quarry sites in Mpape, Abuja, Nigeria. Journal of Remote Sensing \& GIS. 